\title{
Hepatoprotective and antioxidant activities of extracts from Salvia-Nelumbinis naturalis against nonalcoholic steatohepatitis induced by methionine- and choline-deficient diet in mice
}

Yang Liu ${ }^{1 \dagger}$, Haiyan Song ${ }^{1 \dagger}$, Lei Wang ${ }^{2}$, Hanchen Xu' ${ }^{1}$ Xiangbing Shu' ${ }^{1}$ Li Zhang ${ }^{1}$, Ying Li ${ }^{2}$, Dongfei $\mathrm{Li}^{3}$ and Guang $\mathrm{Ji}^{\mathrm{i}^{*}}$

\begin{abstract}
Background: Nonalcoholic steatohepatitis (NASH), the advanced stage of nonalcoholic fatty liver disease that is characterized by both steatosis and severe injury in liver, still lacks efficient treatment. The traditional Chinese formula Salvia-Nelumbinis naturalis (SNN) is effectively applied to improve the symptoms of nonalcoholic simple fatty liver (NAFL) patients. Previous studies have confirmed that SNN could reduce the liver lipid deposition and serum transaminases of NAFL experimental models. This study aims to determine whether SNN is effective for murine NASH model and investigate the underlying pharmacological mechanisms.
\end{abstract}

Methods: C57BL/6 J mice were fed with methionine- and choline-deficient (MCD) diet for six weeks to induce NASH. Simultaneously, SNN or saline was intragastrically administered daily to the mice in the SNN or model group, respectively. A standard diet was given to the control mice. Serum biochemical indices and tumor necrosis factor-a were measured. Liver histopathology was observed, and the contents of triglycerides and lipid peroxide malondialdehyde (MDA) in liver homogenates were evaluated. The hepatic expression and/or activation of genes associated with inflammation, apoptosis, and oxidative stress were determined by quantitative RT-PCR or Western blot analysis.

Results: The prominent liver steatosis displayed in the NASH model was prevented by SNN. The liver injury of NASH mice was obviously manifested by the increased levels of serum transaminases and bilirubin, as well as the lobular inflammation, elevated pro-inflammatory cytokines, and upregulated apoptosis in liver tissues. SNN administration improved the aforementioned pathological changes. The increased hepatic levels of MDA and cytochrome P450 2E1 of the model confirmed the unregulated balance of oxidative stress. The hepatic expression of nuclear factor erythroid 2-related factor 2 and its target genes decreased, whereas c-Jun $\mathrm{N}$-terminal kinase activation in the model mice increased. Treating the mice with SNN significantly improved oxidative stress-related harmful factors.

Conclusions: This study shows that SNN can protect the liver from severe steatosis and damage induced by MCD diet, which suggests the potential use of SNN on the treatment of NASH patient. The results also indicate that improving the hepatic antioxidant capability of the liver may contribute to the underlying hepatoprotective mechanism.

Keywords: Salvia-Nelumbinis naturalis, NASH, Liver injury, Oxidative stress

\footnotetext{
*Correspondence: jiliver@vip.sina.com

${ }^{\dagger}$ Equal contributors

'Institute of Digestive Diseases, Longhua Hospital, Shanghai University of

Traditional Chinese Medicine, Shanghai 200032, China

Full list of author information is available at the end of the article
}

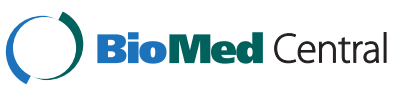

(c) 2014 Liu et al.; licensee BioMed Central Ltd. This is an Open Access article distributed under the terms of the Creative Commons Attribution License (http://creativecommons.org/licenses/by/4.0), which permits unrestricted use, distribution, and reproduction in any medium, provided the original work is properly credited. The Creative Commons Public Domain Dedication waiver (http://creativecommons.org/publicdomain/zero/1.0/) applies to the data made available in this article unless otherwise stated. 


\section{Background}

Nonalcoholic fatty liver disease (NAFLD) is an increasing prevalent health problem that ranges from simple fatty infiltration of the liver parenchyma (nonalcoholic simple fatty liver, NAFL) to steatosis with inflammation and hepatocellular ballooning (nonalcoholic steatohepatitis, NASH) and ultimately cirrhosis [1]. Patients with NAFL appear to have a nonprogressive course with benign prognosis. However, approximately $10 \%$ to $30 \%$ of NAFL patients develop NASH with more serious form of liver damage, which may further progress to cirrhosis in as many as $25 \%$ of the cases and suffer from its complications, including portal hypertension, liver failure and hepatocellular carcinoma [2].

NAFLD begins with an aberrant lipid accumulation in the liver [3], followed by initial changes that induce the liver to be sensitive to oxidative stress (OS) and proinflammatory cytokines [4]. OS is an etiological factor in many acute and chronic liver diseases and plays a critical role in the progression of NAFL to NASH [5]. Sources of OS include elevated level of cytochrome P450 2E1 (CYP2E1), lipid peroxidation, and cytokine induction $[6,7]$. CYP2E1 is an inducible enzyme hydroxylating fatty acid that can initiate lipid peroxidation process $[8,9]$. CYP2E1 is frequently induced in NASH, leading to increased reactive oxygen species (ROS) that trigger OS [8]. Considerable OS causes lipid peroxidation of cell membrane and activation of hepatic stellate cell, resulting in inflammation, apoptosis, and fibrogenesis [10]. Increased OS can enhance the secretion of inflammatory cytokines and hepatocyte apoptosis, which possess pivotal roles in the pathogenesis of liver damage in NASH [11-13]. Pro-inflammatory cytokines, such as tumor necrosis factor- $\alpha$ (TNF- $\alpha$ ) and interleukin-1 $\beta$ (IL-1 $\beta$ ), which are involved in liver injury and repair, are also considered as NASH indicators [14-17]. TNF- $\alpha$ plays an important role throughout the progression of steatosis to NASH [12]. The effects of TNF- $\alpha$ result in additional lipid peroxidation of mitochondrial membranes, thereby further enhancing OS in NASH [18]. Therefore, inhibiting TNF- $\alpha$ can suppress liver injury in NASH [19].

Valuable therapeutic interventions against NASH remain limited; hence, searching for a safe and effective drug is relatively essential [20]. Salvia-Nelumbinis naturalis (SNN) formula (initially called Jiang Zhi Granule) is a compound prescription in traditional Chinese medicine composed of five medicinal herbs, namely, Salviae (Danshen in China), Nelumbinis (Heye in China), Herba Gynostemmatis (Jiaogulan in China), Rhizoma Polygoni Cuspidati ( Huzhang in China), and Herba Artemisiae scopariae (Yinchen in China) [21,22]. This complex prescription has been used to treat NAFL in clinical practice in China, with significant effects of alleviating hepatic steatosis with few side effects [23]. In 2008, SNN was granted the permission and certification by the Chinese SFDA (No. 2008 L11181) to be used as drug for clinical trials. $\mathrm{SNN}$ can improve the liver to spleen ratio (L/S ratio) and decrease the body mass index of NAFL patients [23]. Previous in vivo studies have also confirmed the effect of SNN on NAFL and partially unraveled its underlying mechanisms, including improving leptin and insulin resistance, inhibiting transcription of liver $\mathrm{X}$ receptor $\alpha(\mathrm{LXR}-\alpha)$-mediated sterol regulatory element binding protein-1c (SREBP-1c), and maturation of SREBP-1c independent of LXR- $\alpha$ [24-27]. In vitro studies have indicated that the components extracted from SNN reduce lipid droplet deposition and increase the resistance to damage of hepatocytes induced by free fatty acids $[21,28]$. Taken together, our previous studies mainly focused on the therapeutic function of SNN on simple steatosis. Therefore, in the present study, we tried to determine whether and how SNN is potentially effective for NASH. For this objective, the NASH model of mouse induced by methionine- and choline-deficient (MCD) diet was used, of which the major pathological factors are excess OS and failure of lipid transport from hepatocytes [29]. SNN extract was administered to the NASH model to observe the efficacy and to further investigate the related molecular mechanism of the traditional formula.

\section{Methods}

\section{Experimental animals}

Male C57BL/6 J mice weighing $20 \mathrm{~g}$ to $25 \mathrm{~g}$ were purchased from Shanghai SLAC Laboratory Animal Technology Company (License No. SCXK (HU) 2007-0005). The animals were housed in a standard $12 \mathrm{~h}$ light/dark cycle at $22 \pm 2^{\circ} \mathrm{C}$ with $55 \pm 10 \%$ humidity and had access to food and water. The animal experiments were approved by the Institutional Animal Care and Use Committee of Shanghai University of Traditional Chinese Medicine. All animal procedures were performed in accordance to the guide for the care and use of laboratory animals [30].

\section{Drug and chemicals}

SNN was provided by the Department of Pharmacy of Longhua Hospital, Shanghai, China. This herbal formula was triturated and blended to powder. Subsequently, $20 \mathrm{mg}$ of the powder was extracted with $25 \mathrm{~mL}$ of water/methanol $(5: 95, \mathrm{~V} / \mathrm{V})$ in an ultrasonic bath at $42 \mathrm{kHz}$ frequency for 30 min. The chemical profile of SNN has already been analyzed by ultra-performance liquid chromatography [28]. SNN was stored at $4^{\circ} \mathrm{C}$ and diluted to the desired concentrations in distilled water at the time of administration. Hematoxylin-eosin (HE) solution was purchased from Yixin Biological Technology, Inc. (Shanghai, China), and Oil red $\mathrm{O}$ was from Sigma-Aldrich (St. Louis, MO, USA). All reagents were at least of analytic grade and applied according to manufacturer's instructions. 


\section{Experimental design of animals}

A total of 36 C57BL/6 J mice were adaptively fed for 7 days and were randomly allocated into the following experimental groups ( $\mathrm{n}=12$ per group) according to their body weights: (1) the control mice given a standard control diet, (2) the model mice fed with MCD diet, and (3) mice fed with MCD diet together with oral garvage of SNN (860 mg/kg body weight) once a day. The application dose of SNN was calculated based on the standard dose in clinical practice, previous in vivo experiments, dose conversion among animals and human, and extract yield $[22,25,26,28]$. The MCD and control diets were purchased from Research Diets, Inc. (New Brunswick, NJ, USA). At the end of the sixth week, all animals were fasted overnight and sacrificed. The serum was separated for further investigation. Mice livers were weighed and frozen or fixed in $10 \%$ formalin.

\section{Serum biochemical analysis}

The alanine aminotransferase activity (ALT), aspartate aminotransferase activity (AST), total bilirubin (TBIL), total cholesterol (TC), triglycerides (TG), high-density lipoprotein cholesterol (HDL-c), and low-density lipoprotein cholesterol (LDL-c) were determined to assess liver function using commercially available kits (Jiancheng Institute of Bio Engineering, Inc., Nanjing, China) according to the manufacturer's instructions. The serum concentration of TNF- $\alpha$ was measured by a Bio-Plex assay kit (Bio-Rad, Hercules, USA) with the Bio-Plex 200 system (Bio-Rad).

\section{Histological examination}

The liver sections were stained with HE according to the standard methods. In brief, the fresh liver tissue samples were fixed in $10 \%$ formalin and embedded in paraffin. The samples were cross-cut into slices of $4 \mu \mathrm{m}$ to $5 \mu \mathrm{m}$ and stained with HE staining solution. Finally, the stained sections were observed and photographed under a light microscope (with 200x magnification). All HE stained sections $(n=12)$ were evaluated in a blinded manner by two pathologists for NAFLD activity score (NAS) including the components of steatosis, inflammation and hepatocyte ballooning. The scoring system was as follows: steatosis grade $(0-3 ; 0:<5 \%, 1: 5 \%-33 \%, 2$ : $33 \%-66 \%$, and $3:>66 \%)$, lobular inflammation $(0-3 ; 0$ : no foci, 1 : few foci $/ 200 \times, 2$ : many foci $/ 200 \times$, and $3:>4$ foci/200x scope), and balooning (0-2; 0 : no foci, $1:<2$ foci/200x, and 2: $2-4$ foci/200× [31].

\section{Oil Red O staining}

For Oil Red O staining, a stock solution of Oil Red O $(0.5 \mathrm{~g} / 100 \mathrm{~mL})$ in isopropanol was prepared, and protected from light. After being fixed with $10 \%$ paraformaldehyde for $30 \mathrm{~min}$, the frozen liver sections with $10 \mu \mathrm{m}$ thickness were stained with Oil Red O for 60 min. Nonspecific staining was removed with $70 \%$ ethanol. The sections were counterstained with hematoxylin diluted at 1:10 and mounted with $80 \%$ glycerol. The stained sections were visualized and photographed under a microscope (Olympus IX71, Tokyo, Japan).

\section{Evaluation of lipid content in liver tissues}

Exactly $3 \mathrm{ml}$ of Ethanol-acetone (1:1) was added to the hepatic tissues $(200 \mathrm{mg})$, which was then homogenized in an ice bath and mixed thoroughly at $4^{\circ} \mathrm{C}$ overnight. After $24 \mathrm{~h}$, the liver tissues were centrifuged at $3000 \mathrm{rpm}$ and $4^{\circ} \mathrm{C}$ for $20 \mathrm{~min}$. Subsequently, the supernatant was transferred to a new tube, and TG was measured based on the instructions on TG assay kit (Jiancheng Institute of Bio Engineering, Inc.) using the colorimetric method.

\section{Measurement of MDA in liver tissues}

The contents of malondialdehyde (MDA) in the liver tissues were determined through thiobarbutaric acid (TBA) method using a MDA testing kit (Beyotime Institute of Biotechnology, shanghai, China). Briefly, the liver tissues (200 mg) were homogenized in $0.15 \mathrm{M} \mathrm{KCl}$ solution. The homogenate and $10 \%$ trichloroacetic acid were mixed (1:1) and centrifuged. The supernatant was suspended into TBA. After placing in a water bath for $15 \mathrm{~min}$, the samples were centrifuged at $1000 \mathrm{~g}$ for $15 \mathrm{~min}$. The optical density of the supernatants was measured at $532 \mathrm{~nm}$ with a BioTek SynergyH4 enzyme-labeled instrument (USA).

\section{Quantitative reverse transcription polymerase chain reaction (RT-PCR)}

The changes of different genes in the liver tissues of MCD mice were validated by conducting quantitative RT-PCR. The total RNA was converted to cDNA by using reverse transcription kits (Promega, Madison, WI, USA). All primers were synthesized by Shanghai Shine Gene Company. The sequences of the primers used in this study are indicated in Table 1. Quantitative RT-PCR was then performed using a SYBR Green PCR Master Mix kit (Applied Biosystems, Carlsbad, CA, USA) according to the manufacturer's protocol. Amplification of $\beta$-actin was performed in parallel as a relatively invariant internal reference. The $2^{-\Delta \Delta \mathrm{Ct}}$ method was applied for data analysis.

\section{Western blot}

Protein concentration was determined using a BCA protein assay kit (CoWin Bioscience, Beijing, China). The protein sample was resolved by $10 \%$ denaturing sodium dodecyl sulfate polyacrylamide gel electrophoresis and was blotted onto Immobilon-P PVDF membranes (Millipore, Billerica, MA, USA). After being blocked by $5 \%$ milk, the membrane was incubated with primary antibodies at $4{ }^{\circ} \mathrm{C}$ 


\begin{tabular}{|c|c|}
\hline Gene & Primer sequence \\
\hline \multirow[t]{2}{*}{$\beta$-actin } & Forward : 5'-GAGACCTTCAACACCCCAGC-3' \\
\hline & Reverse : 5'-ATGTCACGCACGATTTCCC-3' \\
\hline \multirow[t]{2}{*}{ TNF-a } & Forward: 5'-CCCTCCAGAAAAGACACCATG-3' \\
\hline & Reverse : 5'-CACCCCGAAGTTCAGTAGACAG-3' \\
\hline \multirow[t]{2}{*}{$I L-1 \beta$} & Forward: 5'-TCGTGCTGTCGGACCCAT-3' \\
\hline & Reverse : 5'-GGCTTGTGCTCTGCTTGTGA-3' \\
\hline \multirow[t]{2}{*}{ CYP2E1 } & Forward : 5'-AGGCTGTCAAGGAGGTGCTAC-3' \\
\hline & Reverse : 5'-GTTTCCCCATTCCCCAGTC-3' \\
\hline \multirow[t]{2}{*}{ Nrf2 } & Forward : 5'-TCTTCCATTTACGGAGACCCA-3' \\
\hline & Reverse : 5'-GATTCACGCATAGGAGCACTG-3' \\
\hline \multirow[t]{2}{*}{ Nqo1 } & Forward: 5'-TCAACTGGTTTACAGCATTGGC-3' \\
\hline & Reverse : 5'-GCTTGGAGCAAAATAGAGTGGG-3' \\
\hline \multirow[t]{2}{*}{ Gstp1 } & Forward : 5'-ACCCTGCTGTCCCAGAACC-3' \\
\hline & Reverse : 5'-GCGAGCCACATAGGCAGAG-3' \\
\hline
\end{tabular}

overnight. The antibodies against c-Jun N-terminal kinase (JNK), Phospho-JNK, activated Caspase-3, Bcl2-associated X (Bax) protein, and Bcl-2 were purchased from Cell Signaling Technology (Danvers, MA, USA). The antibody against CYP2E1 was obtained from Abcam (Cambridge, MA, USA), and $\beta$-actin was obtained from Huaan Biological Technology (Hangzhou, China). Subsequently, the membrane was incubated in a horseradish peroxidase-conjugated goat anti-rabbit or anti-mouse secondary antibody from Thermo Scientific (Rockford, IL, USA) for $1 \mathrm{~h}$. The signal was visualized by enhanced chemiluminescence HRP substrate (Millipore, Billerica, MA, USA) and acquired by GBOX Chemi XT4 System (Syngene, Cambridge, UK). GeneTools software (Syngene) was used for quantification.

\section{Statistical analysis}

The data were expressed as mean \pm standard deviation. Statistical analyses were carried out using one-way ANOVA, followed by Tukey's post-hoc test to assess the differences between the two groups. The data from qPCR, Western blot and pathological scores were compared using Kruskal-Wallis ANOVA test followed by Dunn's multiple comparison test. SPSS 18.0 was used for all statistical analyses. $P<0.05$ was considered statistically significant.

\section{Results}

SNN reversed the changes in body and liver weights and serum lipid of the NASH model

The body and liver weights of mice were measured at the end of the experiment. In contrast to those of the control group, the body weight, liver weight, and liver-body weight ratio remarkably decreased in the NASH model
$(P<0.001)$. These parameters significantly improved in mice treated with $\mathrm{SNN}(P<0.001)$ (Figure $1 \mathrm{~A})$.

Statistically significant differences were also observed in the blood lipid content. The serum levels of TG, TC, HDL-c, and LDL-c decreased in the model group $(P<0.001)$, which were prevented by SNN intervention to a certain extent, particularly the levels of TC, HDL-c $(P<0.01)$, and LDL-c $(P<0.05)$ (Figure 1B).

\section{SNN ameliorated hepatosteatosis induced by MCD diet}

The MCD-induced NASH model was established after the mice were fed with MCD diet for six weeks. Figure 2A shows that the liver sections of the model mice stained with $\mathrm{HE}$ and Oil Red $\mathrm{O}$ displayed abundant accumulation of fat droplets in hepatocytes, together with scattered inflammatory cell infiltration and hepatocellular ballooning degeneration, which were all improved in mice treated with SNN. The steatosis grade of the liver tissue was higher in the model group than in the control group $(P<0.001)$ but was significantly downregulated in the SNN group $(P<0.05)$ (Table 2$)$. The increased liver TG content $(P<0.001)$, which was another proof of lipid deposition in the livers of model mice, was consistently reduced by SNN $(P<0.05)$ (Figure $2 \mathrm{~B})$.

\section{SNN attenuated liver injury induced by MCD diet}

Liver damage was exhibited by the elevated serum values of $\operatorname{ALT}(P<0.01)$, AST $(P<0.01)$, and TBIL $(P<0.001)$ in the model group compared with the control group. SNN treatment prevented the changes to a certain extent (Figure 3A). Inflammatory cytokines were implicated in the pathogenesis of NASH. The MCD diet increased the serum level of TNF- $\alpha(P<0.001)$ and hepatic TNF- $\alpha$ expression $(P<0.05)$ in the model group, which was blocked by SNN (Figure 3B, C). The direct evidence of liver injury was determined by lobular inflammation and hepatocyte death in liver sections. The lobular inflammatory grade $(P<0.001)$, ballooning grade $(P<0.01)$ and the aggregate NAS $(P<0.001)$ were higher in the model mice but was downregulated in the SNN group (Table 2). The increased level of active caspase-3 $(P<0.001)$ indicated more apoptosis of the hepatocytes in MCD dietfed mice than in control mice. This phenomenon was attenuated by SNN intervention $(P<0.01)$. Compared with that in the controlled mice, the anti-apoptosis molecule Bcl-2 expression was reduced $(P<0.05)$ in the model mice but was upregulated in the SNN group $(P<0.05)$. The pro-apoptosis molecule Bax exhibited the converse changing tendency (Figure 3D).

\section{SNN regulated hepatic oxidative stress in MCD diet-induced NASH}

This study also found that SNN could improve the state of OS. The levels of lipid peroxidation (MDA) were 

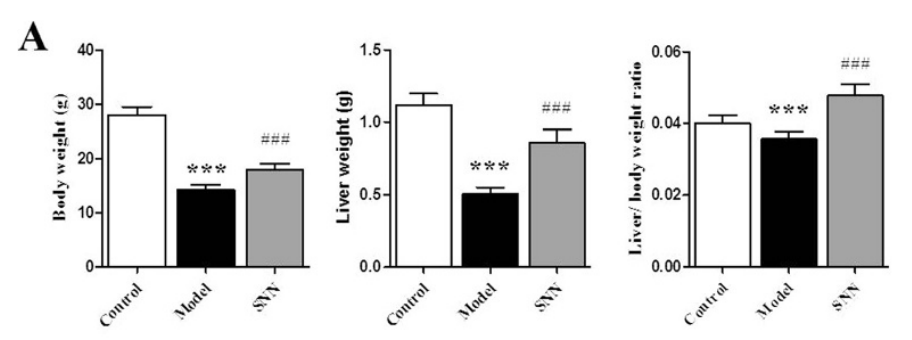

$$
\begin{aligned}
& \square \text { Control } \\
& \text { 亭 } \\
& \text { sodel }
\end{aligned}
$$
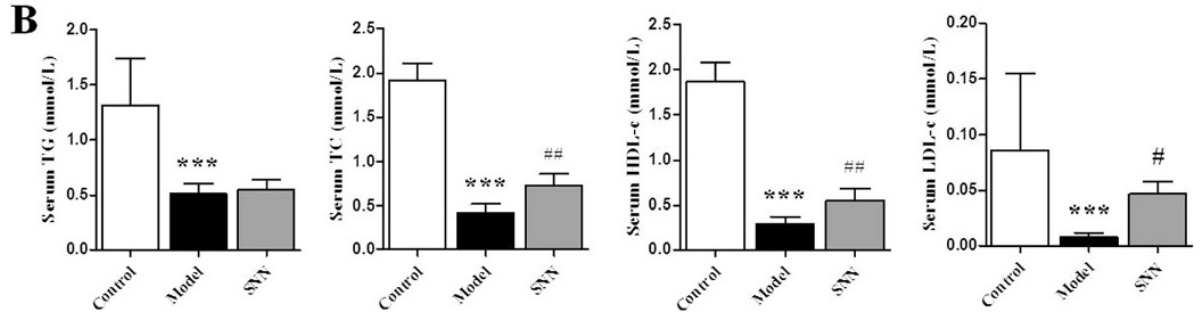

Figure 1 Changes in body and liver weight and serum lipid of mice. (A) Body weight, liver weight, and liver-body weight ratio ( $\mathrm{n}=12$ per group). (B) Serum TG, TC, HDL-C, and LDL-C ( $n=8$ per group). Data are expressed as mean \pm SD, ${ }^{* * *} P<0.001$ vs. control; ${ }^{\# \# \#} P<0.001,{ }^{\# \#} P<0.01$ vs. model.

significantly increased in MCD diet-fed mice compared with those in the control $(P<0.05)$ but were decreased by SNN treatment $(P<0.05)$ (Figure $4 \mathrm{~A})$. The mRNA level of CYP2E1 was not remarkably different among the groups, but its protein level was increased in the model mice and was downregulated by SNN $(P<0.05)$
(Figure 4B, C). After the sixth week, the levels of nuclear factor erythroid 2-related factor 2 (Nrf2) and its target genes, Nqo1 and Gstp1, decreased in the MCD diet-induced NASH model $(P<0.05 v s$. control $)$ but increased in mice administered with SNN $(P<0.05$ vs. model) (Figure 4B). JNK is reported as the bridge

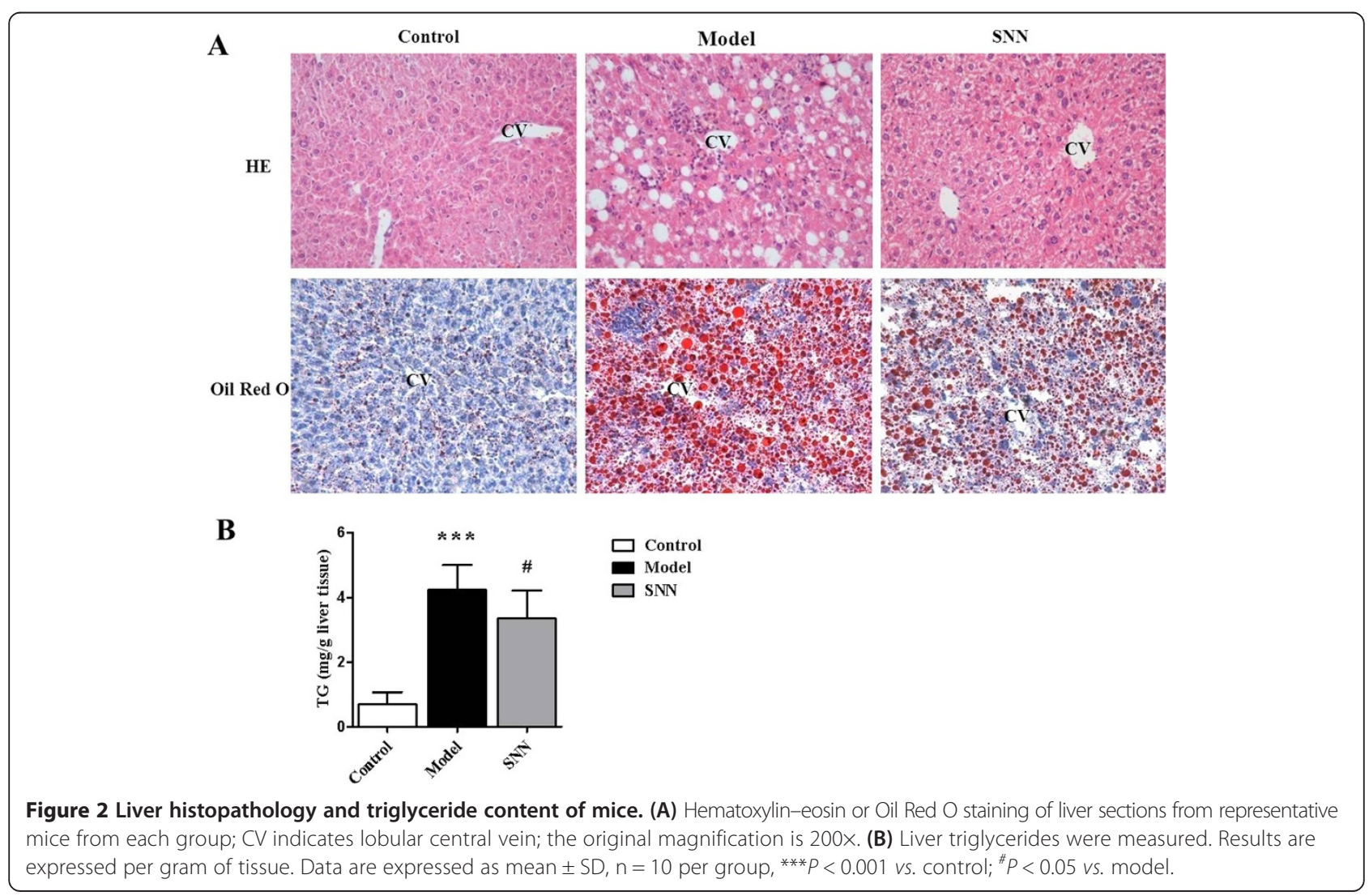


Table 2 Liver histology evaluated by NAFLD Activity score

\begin{tabular}{llll}
\hline Group & Control & Model & SNN \\
\hline Steatosis grade & $0 \pm 0$ & $2.6 \pm 0.52^{* * *}$ & $0.75 \pm 0.71^{\#}$ \\
Lobular inflammatory grade & $0 \pm 0$ & $2.0 \pm 0.76^{* * *}$ & $0.5 \pm 0.53^{\#}$ \\
Hepatocellular balooning & $0 \pm 0$ & $1.4 \pm 0.92^{* *}$ & $0.6 \pm 0.52$ \\
NAS & $0 \pm 0$ & $4.8 \pm 1.90^{* * *}$ & $1.9 \pm 0.79^{\#}$ \\
\hline
\end{tabular}

Data are expressed as mean $\pm S D, n=12$ per group, ${ }^{* * * P}<0.001,{ }^{* * P}<0.01$ vs. control; ${ }^{\#} P<0.05$ vs. model.

molecule of OS and apoptosis [32]. No apparent difference in JNK expression was observed among various groups. However, the level of its active form (p-JNK) increased more than fourfold $(P<0.01)$ in the model mice and was reduced in the SNN group $(P<0.05)$ (Figure $4 \mathrm{C}$ ).

\section{Discussion}

NASH progression is mediated by an inflammatory process in the liver with concomitant tissue damage.
MCD diet-induced model is considered convenient for examining the role of OS and inflammation in the progression of NAFLD [33-35]. Choline and methionine are essential components for very-low-density lipoprotein (VLDL) and lipid oxidation. The deficiency of these components can reduce lipid oxidation and lipid transport outside of the hepatocytes, resulting in lipid accumulation in the liver. Choline and methionine deficiency also causes loss of active methyl which is important for glutathione cycle, leading to decreased antioxidants and promotion of OS [36,37]. The mice fed with MCD diet lost weight because of reduced caloric intake, which is in contrast to humans with NASH who are mostly obese. Thus, differences exist between MCD dietary mouse model and human NASH [37]. However, the MCD NASH model is one of the best established and commonly used dietary models for studying the evolution of inflammation and OS changes associated with NASH [38-40]. Liver injury and OS rapidly occur in MCD diet-
A

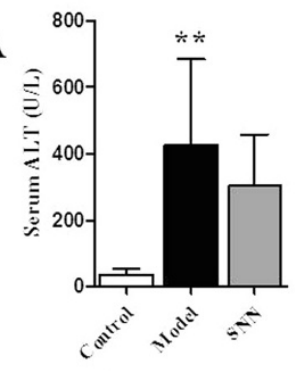

B
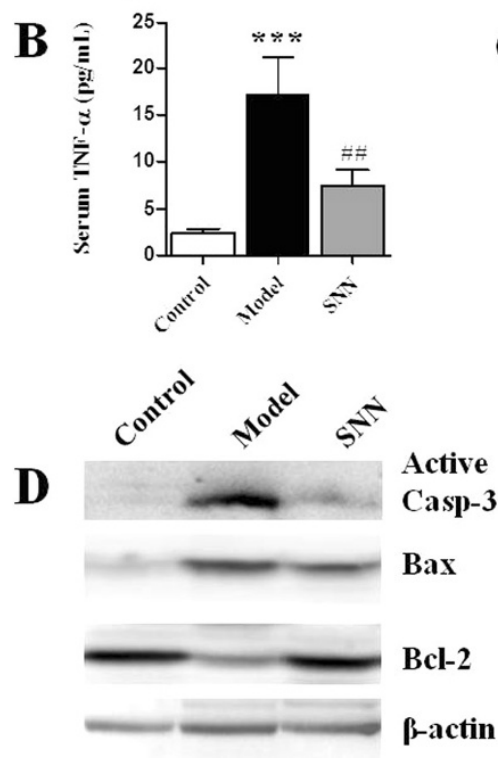
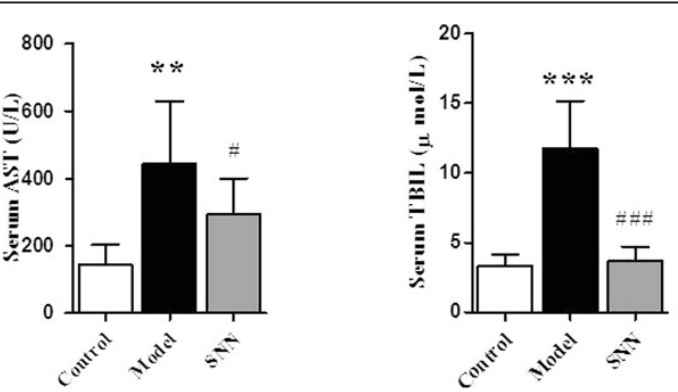

C
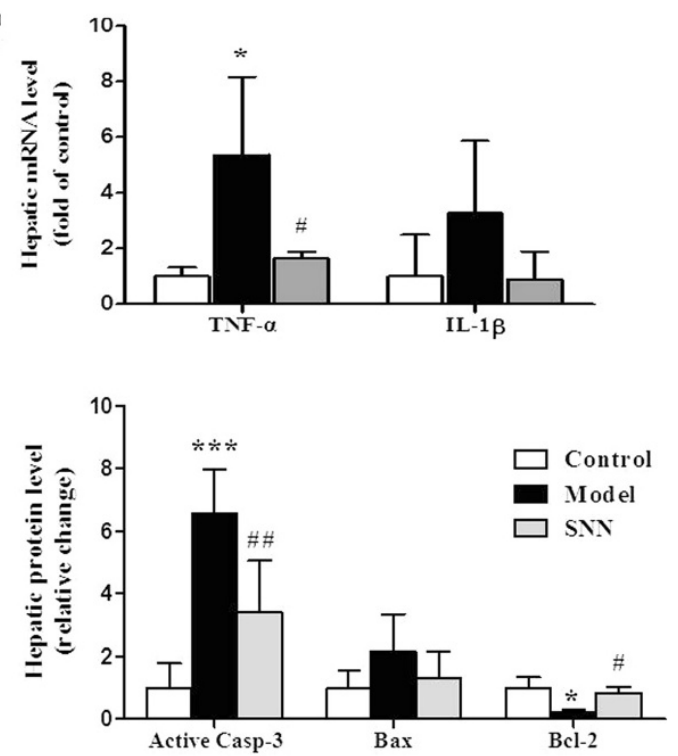

Figure 3 Liver injury-related parameters of mice. (A) Serum ALT, AST and TBIL were measured ( $n=8$ per group). (B) Serum TNF-a level was measured by Bio-Plex 200 system with Luminex system after labeling with specific Luminex magnetic beads $(n=8$ per group). (C) Hepatic mRNA expression of the liver pro-inflammatory cytokines TNF- $\alpha$ and IL-1 $\beta$ was evaluated by quantitative real-time PCR analysis. Results were normalized to $\beta$-actin expression ( $n=4$ per group). (D) The expression of apoptosis-related genes including active caspase-3, BAX, and Bcl-2 was evaluated by immunoblot analysis of livers from four mice per group. $\beta$-actin was determined as the loading control. Data are expressed as mean $\pm S D$, ${ }^{* * *} P<0.001$, ${ }^{* *} P<0.01$, ${ }^{*} P<0.05$ vs. control; ${ }^{\# \# \#} P<0.001,{ }^{\# \#} P<0.01,{ }^{\#} P<0.05$ vs. model. 
A

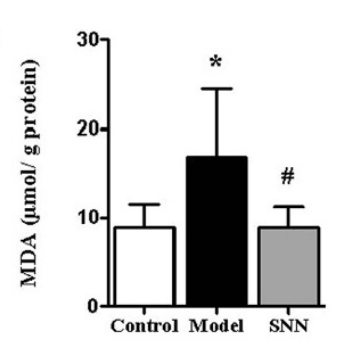

C

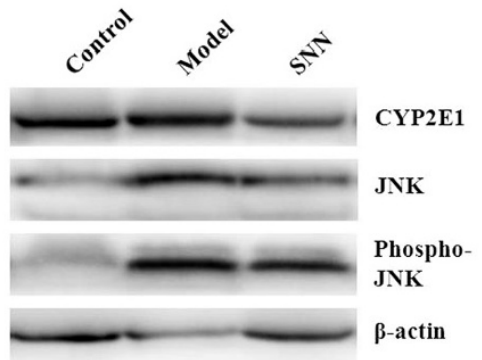

B
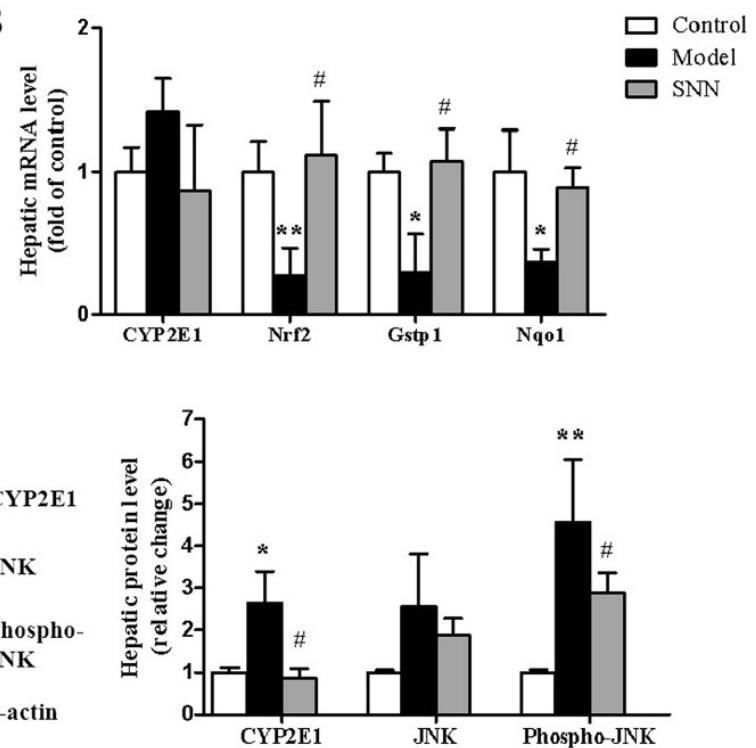

Figure 4 Parameters of oxidative stress of mice. (A) The MDA content of liver was measured. Results are expressed per gram of liver protein extract ( $n=8$ per group). (B) The hepatic mRNA expression of liver oxidant stress related genes including CYP2E1, Nrf2, Gstp1 and Nqo1 was evaluated by quantitative real-time PCR analysis. Results were normalized to $\beta$-actin expression ( $n=4$ per group). (C) The expression of CYP2E1, JNK and phospho-JNK was evaluated by immunoblot analysis of livers from four mice per group. $\beta$-actin was determined as the loading control. Data are expressed as mean $\pm \mathrm{SD},{ }^{* *} P<0.001,{ }^{* *} P<0.01,{ }^{*} P<0.05$ vs. control; ${ }^{*} P<0.05$ vs. model.

fed model compared with other nutritional models. In particular, severe pericentral steatosis may develop in MCD diet-fed model after one week to two weeks; necro-inflammation may occur after two weeks; progressive pericellular and pericentral fibrosis may appear after eight weeks; and markedly enhanced OS can be observed from three weeks of MCD diet. Moreover, the liver injury induced by MCD diet is similar to human NASH $[33,36,37]$. In this study, MCD diet was used to feed C57BL/6 J mice for six weeks. Compared with the mice fed with control diet, hepatic steatosis appeared in the liver tissue accompanied with hepatocellular ballooning and scattered inflammatory cell infiltration, and transaminase activities were significantly increased in the MCD diet-fed mice, which indicate the establishment of the NASH model.

This study demonstrated the strong effect of SNN on preventing NASH development by simultaneously administering MCD diet to mice. The mice fed with MCD diet and administrated with SNN for six weeks exhibited only mild lipid deposition in their livers, instead of the prevalent hepatic steatosis, inflammation and hepatocellular ballooning degeneration that occurred in the NASH model without intervention. Consistently, the hepatocellular injury indicated by the increased serum levels of ALT, AST, bilirubin, and TNF- $\alpha$ and cell apoptosis in the model mice were evidently alleviated in the mice of the SNN group. Compared with our previous studies [22,25-27], the current study elucidated that SNN not only ameliorated steatosis but also improved the more serious liver injury in NASH. In addition, SNN blocked the excessive loss of body and liver weights and decreased the circulating lipid, which occurred in the NASH models. This function is similar to preventing a body from progressing into an unhealthy status.

Our previous studies identified insulin resistance, leptin resistance, and upregulated free fatty acid (FFA) synthesis as parts of the mechanisms involved in the effect of SNN [24-27]. The model mice in the present study showed lower serum levels of insulin and leptin, as well as hepatic FFA synthetases, such as SREBP-1c and stearol-CoA desaturase 1, than those of the control mice (data not shown). By contrast, obviously increased levels of MDA and CYP2E1 were observed in the livers of the NASH mice induced by MCD diet, as reported in previous studies [34,40]. Thus, the present study further focused on whether SNN could improve NASH through regulating OS.

OS is a disturbance between the pro-oxidant/antioxidant balance in favor of the former [41]. As an etiological factor in NASH [42], OS can initiate hepatocellular injury and secondary recruitment of inflammation $[5,8]$. Understanding the pathophysiological mechanisms of OS helps in the development of NASH treatment [43]. MDA is a lipid oxidant that is widely used as a marker of lipid oxidation. MDA increased in $90 \%$ of NASH patients, illustrating the increase of OS $[42,44,45]$. Generation of lipid peroxides results in subsequent damage to hepatic membranes, 
proteins, and DNA. CYP2E1 is also regarded as a common marker of OS. CYP2E1 can oxidize a variety of small molecule substrate. Superoxide anion, a byproduct of the CYP2E1-mediated metabolism, is a very potent ROS, which may serve as part of the second hit to advance the severity of NAFLD to NASH $[46,47]$. The results of this study showed increased CYP2E1 and MDA in the hepatic tissue of model mice, indicating the enhancement of OS. Nrf2 serves as a master regulator of a cellular defense system against OS. Upon exposure to ROS, Nrf2 dissociates and translocates into the nucleus, in which it binds to cis-acting antioxidant-responsive elements (ARE) and promotes the transcription of cytoprotective genes, such as $\mathrm{NAD}(\mathrm{P}) \mathrm{H}$-quinone oxidoreductase (Nqo) and glutathioneS-transferase (Gst) [48]. Increased expression of Nrf2 has been reported in mice fed with MCD diet for two weeks as an adaptive response to elevated ROS [49]. However, in the present study, low levels of Nrf2 and its targeted genes, Nqo1 and Gstp1, were observed in the model mice. This result may be attributed to the exhaustion of compensatory mechanism for excess ROS after the mice were fed with MCD diet for six weeks. Previous study has found that livers of Nrf2 (-/-) mice on the MCD diet suffered more OS than their wild-type counterparts did [50]. The consequences of OS include lipid peroxidation in cell membranes, stellate cell activation in the liver leading to liver fibrosis, chronic inflammation, and apoptosis $[35,43]$. Moreover, ROS overproduction can promote hepatic insulin resistance, which further aggravates fatty liver [43]. Increased CYP2E1 activity correlates with the degree of steatosis and level of the inflammatory cytokines (e.g., TNF- $\alpha$ ) involved in the pathology of NASH [6]. Thus, in the murine MCD diet-induced NASH model, hepatic injury develops as the decreased hepatocellular antioxidant defenses is overwhelmed by OS [43]. The mice of the SNN group demonstrated lower levels of MDA and CYP2E1 than the mice fed with MCD diet but exhibited more expressions of Nrf2, Nqo1, and Gstp1. Thus, administratering SNN helped reverse the OS imbalance. This finding may be attributed to the integrated function of the natural components contained in SNN, such as protopanaxadiol, berberine, and resveratrol. These components are antioxidants that could directly scavenge free radicals or regulate the correlated factors [21,51-53].

Apoptosis is a common mechanism of liver injury. The hepatocyte apoptosis assessed by terminal deoxynucleotidyl transferase dUTP nick end labeling (TUNEL) of liver biopsies and active caspases- 3 and -7 significantly increases in patients with NASH and correlates with disease severity [54]. The mitogen-activated protein kinase JNK signaling pathway contributes to stress-induced apoptosis. Previous studies demonstrated that sustained JNK activation occurs with the development of steatohepatitis, which can be activated by overexpression of CYP2E1 in NASH. The JNK pathway may be a link between OS and apoptosis [32,55]. OS-associated JNK-mediated apoptosis requires the activation of the transcription factor c-Jun or induces apoptosis in a transcription-independent process by activating proapoptotic members of the $\mathrm{Bcl}-2$ family, including Bim and Bax, or inactivating Bcl-2 and Bcl-xL [56,57]. Consistently, this study observed that the activation of caspase-3 and JNK increased in the MCD diet-induced NASH model, together with low expression of Bcl-2. Such finding implies that JNK may mediate apoptosis by regulating Bcl- 2 . SNN treatment significantly increased the level of activated caspase- 3 and JNK and downregulated $\mathrm{Bcl}-2$, suggesting the function of SNN in the regulation of the pathway of OS-JNK apoptosis.

\section{Conclusions}

By administering SNN in the MCD diet-induced murine model, this study demonstrates that SNN has a beneficial effect on inhibiting hepatic steatosis and improving liver injury, including inflammation and hepatocyte apoptosis in NASH. The results of this study suggest the potential application of SNN in treating NAFLD with serious form of liver injury. However, these findings still require further validation by conducting clinical trials. SNN is a complex herbal medicine that may possess multiple pharmaceutical targets; however, in this study, the effect of regulating OS as well as subsequent anti-apoptosis and antiinflammation functions were prominently observed to attenuate liver injury. Such functions contribute to explain the mechanism of SNN in modulating the pathophysiology of NASH. Furthermore, since OS and inflammation are also the major mechanism of alcoholic fatty liver disease $[46,58,59]$, SNN might also have potential for the treatment of this common liver disease.

Competing interests

The authors declare that they have no competing interests.

\section{Authors' contributions}

SH designed the research. $L Y, W L, Z L$ and $L D$ contributed to the animal experiments. LY, XH, SX and LY performed the biochemical and histopathological experiments. SH and LY analyzed the data and wrote the paper. JG conceived of the study and supervised the coordination. Co-first authors: LY and SH. All authors have read and approved the final manuscript.

\section{Acknowledgments}

This study was supported by the Innovation Program of shanghai Municipal Education Commission ( No.12ZZ119, No.E03008) shanghai, China; National Natural Science Foundation of China (No.81202667, No.81273727); Research Grant for Health Science and Technology of Pudong Health Bureau of Shanghai (PW2012A-40), China.

\section{Author details}

${ }^{1}$ Institute of Digestive Diseases, Longhua Hospital, Shanghai University of Traditional Chinese Medicine, Shanghai 200032, China. ${ }^{2}$ Department of Liver Diseases, Longhua Hospital, Shanghai University of Traditional Chinese Medicine, Shanghai 200032, China. ${ }^{3}$ Department of Traditional Chinese Medicine, East Hospital, Tongji University, Shanghai 200120, China. 
Received: 13 August 2014 Accepted: 28 October 2014

Published online: 19 November 2014

\section{References}

1. Ratziu V, Bellentani S, Cortez-Pinto H, Day C, Marchesini G: A position statement on NAFLD/NASH based on the EASL 2009 special conference. J Hepatol 2010, 53:372-384

2. Williams CD, Stengel J, Asike MI, Torres DM, Shaw J, Contreras M, Landt CL, Harrison SA: Prevalence of nonalcoholic fatty liver disease and nonalcoholic steatohepatitis among a largely middle-aged population utilizing ultrasound and liver biopsy: a prospective study. Gastroenterology 2011, 140:124-131.

3. Cohen JC, Horton JD, Hobbs $\mathrm{HH}$ : Human fatty liver disease: old questions and new insights. Science 2011, 332:1519-1523.

4. Petta S, Muratore C, Craxi A: Non-alcoholic fatty liver disease pathogenesis: the present and the future. Dig Liver Dis 2009, 41:615-625.

5. Oliveira CP, da Costa Gayotto LC, Tatai C, Della Bina Bl, Janiszewski M, Lima ES, Abdalla DS, Lopasso FP, Laurindo FR, Laudanna AA: Oxidative stress in the pathogenesis of nonalcoholic fatty liver disease, in rats fed with a choline-deficient diet. J Cell Mol Med 2002, 6:399-406.

6. Aubert J, Begriche K, Knockaert L, Robin MA, Fromenty B: Increased expression of cytochrome P450 2E1 in nonalcoholic fatty liver disease: mechanisms and pathophysiological role. Clin Res Hepatol Gastroenterol 2011, 35:630-637.

7. James O, Day C: Non-alcoholic steatohepatitis: another disease of affluence. Lancet 1999, 353:1634-1636.

8. Robertson G, Leclercq I, Farrell GC: Nonalcoholic steatosis and steatohepatitis. II. Cytochrome P-450 enzymes and oxidative stress. Am J Physiol Gastrointest Liver Physiol 2001, 281:G1135-G1139.

9. Niemela O, Parkkila S, Juvonen RO, Viitala K, Gelboin HV, Pasanen M: Cytochromes P450 2A6, 2E1, and 3A and production of protein-aldehyde adducts in the liver of patients with alcoholic and non-alcoholic liver diseases. J Hepatol 2000, 33:893-901.

10. He J, Hu B, Shi X, Weidert ER, Lu P, Xu M, Huang M, Kelley EE, Xie W: Activation of the aryl hydrocarbon receptor sensitizes mice to nonalcoholic steatohepatitis by deactivating mitochondrial sirtuin deacetylase Sirt3. Mol Cell Biol 2013, 33:2047-2055.

11. Wanless IR, Shiota $K$ : The pathogenesis of nonalcoholic steatohepatitis and other fatty liver diseases: a four-step model including the role of lipid release and hepatic venular obstruction in the progression to cirrhosis. Semin Liver Dis 2004, 24:99-106.

12. Harrison SA, Kadakia S, Lang KA, Schenker S: Nonalcoholic steatohepatitis: what we know in the new millennium. Am J Gastroenterol 2002, 97:2714-2724

13. Cortez-Pinto H, de Moura MC, Day CP: Non-alcoholic steatohepatitis: from cell biology to clinical practice. J Hepatol 2006, 44:197-208.

14. Dinarello CA: The IL-1 family and inflammatory diseases. Clin Exp Rheumatol 2002, 20:S1-S13.

15. Pober JS: Endothelial activation: intracellular signaling pathways. Arthritis Res 2002, 4(Suppl 3):S109-S116

16. Alisi A, Manco M, Devito R, Piemonte F, Nobili V: Endotoxin and plasminogen activator inhibitor-1 serum levels associated with nonalcoholic steatohepatitis in children. J Pediatr Gastroenterol Nutr 2010, 50:645-649.

17. Han YP, Tuan TL, Hughes M, Wu H, Garner WL: Transforming growth factor-beta - and tumor necrosis factor-alpha -mediated induction and proteolytic activation of MMP-9 in human skin. J Biol Chem 2001, 276:22341-22350.

18. Malaguarnera M, Di Rosa M, Nicoletti F, Malaguarnera L: Molecular mechanisms involved in NAFLD progression. J Mol Med 2009, 87:679-695.

19. Popivanova BK, Kitamura K, Wu Y, Kondo T, Kagaya T, Kaneko S, Oshima M, Fujii C, Mukaida N: Blocking TNF-alpha in mice reduces colorectal carcinogenesis associated with chronic colitis. J Clin Invest 2008, 118:560-570.

20. Marcolin E, San-Miguel B, Vallejo D, Tieppo J, Marroni N, Gonzalez-Gallego J, Tunon MJ: Quercetin treatment ameliorates inflammation and fibrosis in mice with nonalcoholic steatohepatitis. J Nutr 2012, 142:1821-1828.

21. Song $H Y$, Zhang L, Pan JL, Yang LL, Ji G: Bioactivity of five components of Chinese herbal formula Jiangzhi granules against hepatocellular steatosis. J Integr Med 2013, 11:262-268.
22. Ma ZS, Liu T, Zheng PY, Xing $L$, Ji G: Effect of Chinese Medicine Jiangzhi Granula on Hepaticlipid of Nonalcoholic Fatty Liver Disease Rats. Chin Arch Tradit Chin Med 2007, 25:942-944.

23. Pan J, Wang M, Song H, Wang L, Ji G: The efficacy and safety of traditional chinese medicine (jiang zhi granule) for nonalcoholic Fatty liver: a multicenter, randomized, placebo-controlled study. Evidence-based Complement Altern Med: eCAM 2013, 2013:965723.

24. Zhang L, Liu T, Wang M, Xing $L$, Ji G, Zheng PY: Effect of Jiang zhi granula on leptin and leptin receptor of hypothalamus in rats with nonalcoholic liver disease. Chin J Integr Tradit West Med Liver Dis 2009, 19:88-91.

25. Zheng PY, Zhang L, Liu T, Wang M, Xing L, Ji G: Effect of Jiangzhi granule on STAT3 expression in hypothalamus of rats with nonalcoholic fatty liver disease. World Chin J Digestol 2009, 17:753-757.

26. Wang $M$, Sun $S$, Wu T, Zhang L, Song $H$, Hao W, Zheng $P$, Xing L, Ji G: Inhibition of LXRalpha/SREBP-1C-Mediated Hepatic Steatosis by Jiang-Zhi Granule. Evidence-based Complement Altern Med: eCAM 2013, 2013:584634.

27. Zhang $L, X u J$, Song $H$, Yao Z, Ji G: Extracts from Salvia-Nelumbinis naturalis alleviate hepatosteatosis via improving hepatic insulin sensitivity. J Trans/ Med 2014, 12:236.

28. Lu YL, Wang M, Zhang L, He YQ, Yang L, Wang CH, Wang ZT, Ji G: Simultaneous determination of six components in the 'Jiang-Zhi' granule by UPLC-MS analysis. Chin J Nat Med 2010, 8:449-455.

29. Leclercq IA, Farrell GC, Field J, Bell DR, Gonzalez FJ, Robertson GR: CYP2E1 and CYP4A as microsomal catalysts of lipid peroxides in murine nonalcoholic steatohepatitis. J Clin Invest 2000, 105:1067-1075.

30. National Research Council (U.S.). Committee for the Update of the Guide for the Care and Use of Laboratory Animals., Institute for Laboratory Animal Research (U.S.), National Academies Press (U.S.): Guide for the care and use of laboratory animals. 8th edn. Washington, D.C.: National Academies Press; 2011.

31. Brunt EM, Kleiner DE, Wilson LA, Belt $P$, Neuschwander-Tetri BA, Network NCR: Nonalcoholic fatty liver disease (NAFLD) activity score and the histopathologic diagnosis in NAFLD: distinct clinicopathologic meanings. Hepatology 2011, 53:810-820.

32. Wang Y, Ausman LM, Russell RM, Greenberg AS, Wang XD: Increased apoptosis in high-fat diet-induced nonalcoholic steatohepatitis in rats is associated with c-Jun $\mathrm{NH} 2$-terminal kinase activation and elevated proapoptotic Bax. J Nutr 2008, 138:1866-1871.

33. Marcolin E, Forgiarini LF, Tieppo J, Dias AS, Freitas LA, Marroni NP: Methionine- and choline-deficient diet induces hepatic changes characteristic of non-alcoholic steatohepatitis. Arq Gastroenterol 2011, 48:72-79.

34. Phung N, Pera N, Farrell G, Leclercq I, Hou JY, George J: Pro-oxidant-mediated hepatic fibrosis and effects of antioxidant intervention in murine dietary steatohepatitis. Int J Mol Med 2009, 24:171-180.

35. George J, Pera N, Phung N, Leclercq I, Yun Hou J, Farrell G: Lipid peroxidation, stellate cell activation and hepatic fibrogenesis in a rat model of chronic steatohepatitis. J Hepatol 2003, 39:756-764.

36. Fan JG, Qiao L: Commonly used animal models of non-alcoholic steatohepatitis. Hepatobiliary Pancreat Dis Int 2009, 8:233-240.

37. Schattenberg JM, Galle PR: Animal models of non-alcoholic steatohepatitis: of mice and man. Dig Dis 2010, 28:247-254.

38. London RM, George J: Pathogenesis of NASH: animal models. Clinics in liver disease 2007, 11:55-74, viii.

39. Min AK, Kim MK, Kim HS, Seo HY, Lee KU, Kim JG, Park KG, Lee IK: Alpha-lipoic acid attenuates methionine choline deficient diet-induced steatohepatitis in C57BL/6 mice. Life Sci 2012, 90:200-205.

40. Park HJ, Han JM, Kim HG, Choi MK, Lee JS, Lee HW, Son CG: Chunggan extract (CGX), methionine-and choline-deficient (MCD) diet-induced hepatosteatosis and oxidative stress in C57BL/6 mice. Hum Exp Toxicol 2013, 32:1258-1269.

41. Sies H: Oxidative stress: from basic research to clinical application. Am J Med 1991, 91:31S-38S.

42. Koruk M, Taysi S, Savas MC, Yilmaz O, Akcay F, Karakok M: Oxidative stress and enzymatic antioxidant status in patients with nonalcoholic steatohepatitis. Ann Clin Lab Sci 2004, 34:57-62.

43. Koek GH, Liedorp PR, Bast A: The role of oxidative stress in non-alcoholic steatohepatitis. Clin Chim Acta 2011, 412:1297-1305.

44. Chalasani N, Deeg MA, Crabb DW: Systemic levels of lipid peroxidation and its metabolic and dietary correlates in patients with nonalcoholic steatohepatitis. Am J Gastroenterol 2004, 99:1497-1502. 
45. Loguercio C, De Girolamo V, de Sio I, Tuccillo C, Ascione A, Baldi F, Budillon G, Cimino L, Di Carlo A, Di Marino MP, Morisco F, Picciotto F, Terracciano L, Vecchione R, Verde V, Del Vecchio Blanco C: Non-alcoholic fatty liver disease in an area of southern Italy: main clinical, histological, and pathophysiological aspects. J Hepatol 2001, 35:568-574.

46. Lieber CS: CYP2E1: from ASH to NASH. Hepatology research: the official journal of the Japan Society of Hepatology 2004, 28:1-11.

47. Abdelmegeed MA, Banerjee A, Yoo SH, Jang S, Gonzalez FJ, Song BJ: Critical role of cytochrome P450 2E1 (CYP2E1) in the development of high fat-induced non-alcoholic steatohepatitis. J Hepatol 2012, 57:860-866

48. Gong P, Cederbaum Al: Nrf2 is increased by CYP2E1 in rodent liver and HepG2 cells and protects against oxidative stress caused by CYP2E1. Hepatology 2006, 43:144-153.

49. Zhang YK, Yeager RL, Tanaka Y, Klaassen CD: Enhanced expression of Nrf2 in mice attenuates the fatty liver produced by a methionine- and choline-deficient diet. Toxicol Appl Pharmacol 2010, 245:326-334.

50. Chowdhry S, Nazmy MH, Meakin PJ, Dinkova-Kostova AT, Walsh SV, Tsujita T, Dillon JF, Ashford ML, Hayes JD: Loss of Nrf2 markedly exacerbates nonalcoholic steatohepatitis. Free Radic Biol Med 2010, 48:357-371.

51. Orsu P, Murthy BV, Akula A: Cerebroprotective potential of resveratrol through anti-oxidant and anti-inflammatory mechanisms in rats. J Neural Transm 2013, 120:1217-1223.

52. Moghaddam HK, Baluchnejadmojarad T, Roghani M, Khaksari M, Norouzi P, Ahooie M, Mahboobi F: Berberine ameliorate oxidative stress and astrogliosis in the hippocampus of STZ-induced diabetic rats. Mol Neurobiol 2014, 49:820-826.

53. Domitrovic R, Jakovac H, Marchesi W, Blazekovic B: Resolution of liver fibrosis by isoquinoline alkaloid berberine in $\mathrm{CCl}(4)$-intoxicated mice is mediated by suppression of oxidative stress and upregulation of MMP-2 expression. J Med Food 2013, 16:518-528.

54. Feldstein $A E$, Canbay A, Angulo P, Taniai M, Burgart $L$, Lindor KD, Gores GJ: Hepatocyte apoptosis and fas expression are prominent features of human nonalcoholic steatohepatitis. Gastroenterology 2003, 125:437-443.

55. Schattenberg JM, Singh R, Wang Y, Lefkowitch JH, Rigoli RM, Scherer PE, Czaja MJ: JNK1 but not JNK2 promotes the development of steatohepatitis in mice. Hepatology 2006, 43:163-172.

56. Yamamoto $\mathrm{K}$, Ichijo $\mathrm{H}$, Korsmeyer $\mathrm{SJ}: \mathrm{BCL}-2$ is phosphorylated and inactivated by an ASK1/Jun N-terminal protein kinase pathway normally activated at G(2)/M. Mol Cell Biol 1999, 19:8469-8478.

57. Wei MC, Zong WX, Cheng EH, Lindsten T, Panoutsakopoulou V, Ross AJ, Roth KA, MacGregor GR, Thompson CB, Korsmeyer SJ: Proapoptotic BAX and BAK: a requisite gateway to mitochondrial dysfunction and death. Science 2001, 292:727-730.

58. Miranda-Mendez A, Lugo-Baruqui A, Armendariz-Borunda J: Molecular basis and current treatment for alcoholic liver disease. Int J Environ Res Public Health 2010, 7:1872-1888.

59. Day CP: Treatment of alcoholic liver disease. Liver Transp/ 2007, 13:S69-S75.

doi:10.1186/s12967-014-0315-x

Cite this article as: Liu et al: Hepatoprotective and antioxidant activities of extracts from Salvia-Nelumbinis naturalis against nonalcoholic steatohepatitis induced by methionine- and choline-deficient diet in mice. Journal of Translational Medicine 2014 12:315.

\section{Submit your next manuscript to BioMed Central and take full advantage of:}

- Convenient online submission

- Thorough peer review

- No space constraints or color figure charges

- Immediate publication on acceptance

- Inclusion in PubMed, CAS, Scopus and Google Scholar

- Research which is freely available for redistribution

Submit your manuscript at www.biomedcentral.com/submit
Ciomed Central 